\title{
CORRELATION EFFECTS IN NUCLEAR TRANSPARENCY
}

\author{
L. L. Frankfurt \\ School of Physics and Astronomy \\ Raymond and Beverly Sackler Faculty of Exact Sciences \\ Tel Aviv University, Ramat Aviv 69978, Israel, Tel Aviv, Israel, \\ on leave of absence from the St.Petersburg Nuclear Physics Institute, Russia \\ E. J. Moniz \\ Center for Theoretical Physics \\ Department of Physics and Laboratory for Nuclear Science \\ Massachusetts Institute of Technology \\ Cambridge, MA 02139 USA \\ and \\ Institute for Theoretical Physics III \\ University of Erlangen-Nürnberg, \\ Erlangen, Germany \\ M. M. Sargsyan \\ School of Physics and Astronomy \\ Raymond and Beverly Sackler Faculty of Exact Sciences \\ Tel Aviv University, Ramat Aviv 69978, Israel, Tel Aviv, Israel, \\ also at the Yerevan Physics Institute, Yerevan, 375036, Armenia \\ M. I. Strikman \\ Pennsylvania State University, University Park, PA 16802, USA, \\ also at the St.Petersburg Nuclear Physics Institute, Russia
}




\begin{abstract}
The Glauber approximation is used to calculate the contribution of nucleon correlations in high-energy $A\left(e, e^{\prime} N\right)$ reactions. When the excitation energy of the residual nucleus is small, the increase of the nuclear transparency due to correlations between the struck nucleon and the other nucleons is mostly compensated by a decrease of the transparency due to the correlations between non detected nucleons. We derive Glauber model predictions for nuclear transparency for the differential cross section when nuclear shell level excitations are measured. The role of correlations in color transparency is briefly discussed.
\end{abstract}

PACS number(s): 25.30.Dh, 25.30.Fj 


\section{INTRODUCTION}

The semiclassical approximation improves with increasing collision energy. This theoretical expectation is supported by the observation that the Glauber approximation describes quite well the recent experimental data on high-energy (e,e'p) reactions [1]. Thus this theoretical framework makes numerous issues in nuclear physics amenable to quantitative study at new facilities, such as CEBAF or HERMES, and provides a baseline for studying color coherent phenomena in the collisions of high energy particles with nuclei. Such phenomena may provide promising new methods of investigation of the nonperturbative and perturbative QCD. One example is the color transparency (CT) phenomenon - suppression of the final state interaction (FSI) in high energy quasielastic large angle reactions with nuclei. Initial motivation for the dominance of small size configurations came from the analysis of the leading perturbative QCD diagrams [2,3] which should dominate at very large momentum transfer. However the recent analysis [4] of realistic models of a nucleon (pion) has found that the electromagnetic form factors of a nucleon and a pion are dominated by smaller than average size configurations already in the nonperturbative domain at $Q^{2} \geq 2-3(\mathrm{GeV} / \mathrm{c})^{2}$. Hence it seems important to study CT in the $A\left(e, e^{\prime} N\right), A\left(e, e^{\prime} N N\right)$ processes at as small $Q^{2}$ as possible. However to achieve these aims one needs both dedicated high resolution experiments at intermediate $Q^{2}$ (see for example [1,5]) and calculations of nuclear transparency within the standard Glauber theory. Such calculations should include nuclear effects such as nucleon correlations in nuclei and the nuclear shell effects.

We approach these problems by adapting the technique developed for high energy hadron scattering off nuclei in the early seventies by Moniz, Nixon and Walecka [6,7] and by Yennie [8]. With the Glauber approximation [9] it is easily shown that nucleon correlations in highenergy coherent hadron-nucleus scattering make a nucleus less transparent. Important point to emphasize is that high-energy particles interact with different nucleons of the nucleus at different moments of time: $t_{1}-t_{2}=c\left(z_{1}-z_{2}\right)$ (that is high-energy processes develop along the light-cone). So the approximation of frozen configurations in the nucleus used 
in the Glauber approximation seems, at first sight, questionable. However, a theoretical analysis based on the light-cone quantum mechanics of nuclei has found [10] that the conventional Glauber formulae can be safely used for description of high-energy processes where contributing nucleon Fermi momenta are not too large. In conventional quantum mechanics, the frozen nucleus approximation emerges from the condition that intermediate nuclear states of importance have excitation energies very small relative to the projectile energy. The application of this technique to the calculation of $\left(e, e^{\prime} p\right)$ cross sections is straightforward though it requires serious modifications due to the different collision geometry. There is no incident hadron, while a fast nucleon is produced in any point of the nucleus so that the final expressions are rather different from those in [6,8]. We will focus on the effects of nucleon correlations for the nuclear transparency in $A\left(e, e^{\prime} N\right)$ reactions at high $Q^{2}$ as well as on the effects of the nuclear shells.

The first question we address is whether correlation effects considered in hadron-nucleus scattering [6,8] are relevant for the propagation of a fast nucleon (produced in a hard scattering) through nuclear matter. The effects of nucleon correlations for nuclear transparency in the $A\left(e, e^{\prime} p\right)$ reaction were considered by several authors [12] [17] in connection with the recent experimental investigations of the color transparency. Benhar et al [12] and Lee and Miller [13] have suggested a modification of the optical model approximation to include the nucleon correlations. They have found that nucleon correlations may significantly (by $\sim 20 \%)$ increase the $\left(e, e^{\prime} p\right)$ cross section and considerably suppress the onset of color transparency $(\mathrm{CT})$ effects especially at intermediate $Q^{2}$. However, in the papers [11, 14, 15] the effects of correlations were estimated to be no more than $5 \%$. We demonstrate here that the role of nucleon correlations depends sensitively on the experimental kinematics. So, we will consider how the shell structure of nuclei observed at medium energies (for a review, see Ref. [18]) should reveal itself in high $Q^{2} A\left(e, e^{\prime} N\right)$ reactions.

To analyze effects of nucleon correlations we consider here the limiting case of coherent final state interactions (FSI), when knocked-out protons rescatter off the residual nucleus coherently and the final state energy of the residual nucleus is fixed and known. A second 
case of incoherent rescattering, when all elastic rescatterings of the proton are allowed and the sum over the final states of residual nucleus is performed, will be discussed elsewhere. The results obtained for coherent final state interactions allow us to calculate nuclear transparency at a fixed value of the ejected nucleon momentum and missing energy. Therefore they could be directly compared with current and planned experiments which have limited momentum and angular acceptance.

The paper is organized as following.

In section 2 we present detailed calculation of the coherent FSI on the basis of the Glauber approximation. By decomposing the ground state wave function over the contribution of two-nucleon correlations we derive the formulae for the description of the $\left(e, e^{\prime} N\right)$ processes in the case of fixed missing energy characterizing particular shells. The deduced formulae take into account the ground and the final nuclear state correlations in a selfconsistent way.

In section 3, formulae obtained in section 2 are used to calculate the nuclear transparency for a proton knocked-out by the virtual photon. Qualitative calculations using a uniform density model of the nucleus, point-like approximation for $N N$ scattering amplitude and $\Theta$ function type of $N N$-correlations allows us to obtain analytic results. Quantitative analysis uses Hartree-Fock single-nucleon wave functions, realistic parameterization of the $N N$ scattering amplitude and correlation effects taken from the current calculations of the nuclear matter. The obtained formulae are extended also to the case when electron produced a small size "nucleon" wave packet which expands while propagating through the nucleus. This gives the possibility of investigating how nucleon correlations influence the onset of color coherent effects in reactions with the excitation of certain shell levels. It is demonstrated that in some cases color transparency may even lead to a decrease of the $\left(e, e^{\prime} N\right)$ cross section with increase of $Q^{2}$. This effect has been considered in Ref. [11, where, however, nucleon correlations were not included in the theoretical analysis.

In section 4 we summarize the basic results of the paper.

The Appendix contains necessary definitions and sum rules for the two nucleon density function. 


\section{COHERENT FINAL STATE INTERACTION}

Within the nonrelativistic theory of nuclei and negleting antisymmetrization of the knocked-out and bound nucleons, the amplitude of $A\left(e, e^{\prime} N\right)$ scattering - $F_{f, 0}$ is given by the formulae:

$$
\begin{aligned}
F_{f 0}^{\gamma^{*} A} & =<\Psi_{\vec{p}_{f}}^{(-)}, \Psi_{f}^{(A-1)}\left|\hat{T}_{\vec{q}}\right| \Psi_{0}^{(A)}> \\
& =\sum_{j} \int d^{3} r_{j} d^{3}\left\{r_{k}\right\} \Psi_{p_{f}}^{(-)}\left(\vec{r}_{j}\right) \Psi_{f}^{(A-1)}\left(\left\{\vec{r}_{k}\right\}\right) T^{e m}\left(Q^{2}\right) e^{i \vec{q}_{j}} \Psi_{0}^{(A)}\left(\vec{r}_{j},\left\{\vec{r}_{k}\right\}\right),
\end{aligned}
$$

where $\Psi_{0}^{(A)}$ is the ground state wave function of nucleus, and $\hat{T}_{\vec{q}} \equiv T^{e m}\left(Q^{2}\right) e^{i \vec{q}_{j}}$ is the one body electromagnetic current operator. In principle, $T^{e m}$ should depend on the presence of other nucleons due to off shell effects; however, in this paper we restrict ourselves to the contribution of nucleons with small Fermi momenta where off shell effects seem to be a small correction. The position of knocked out nucleon $j$ is $\vec{r}_{j} ; \vec{q}$ and $-Q^{2}$ are the three momentum

and mass-square of the virtual photon, $\Psi_{\vec{p}_{f}}^{(-)}$is the wave function of knocked-out nucleon $j$; and $\Psi_{f}^{(A-1)}$ is the wave function of the residual $(A-1)$ nucleus. For simplicity we denote the $\left\{r_{k}\right\} \equiv r_{1}, . . . ., r_{A}$, where the coordinate of knocked-out nucleon is excluded.

It is well known from the low energy studies that the cross section of exclusive $A\left(e, e^{\prime} p\right)$ processes depends strongly on the value of the missing energy $E_{m}$ which characterizes the binding energy of the knocked-out proton, as well as the excitation energy of the residual $(A-1)$ nucleus. If $E_{m}$ is fixed and does not exceed the characteristic value for the nuclear shell excitations $(\lesssim 50 \mathrm{MeV})$ (which is a natural condition for the experiments searching for the color transparency phenomenon) the final state interactions of the knocked-out nucleons with the residual nucleus are dominated by their coherent rescattering off the $(A-1)$-hole residual nucleus.

Neglecting the antisymmetrization between $r_{j} \leftrightarrow\left\{r_{k}\right\}$ the coordinate $r_{j}$ is the knockedout nucleon's coordinate. Then within the impulse approximation for the $\gamma^{*} N$ interaction and Glauber approximation for the interaction of the fast nucleon with the rest of the nucleus, the amplitude $F_{f, 0}$ of the process where a nucleon is knocked-out from a specific 
orbit- $h$, leaving the residual nucleus in a $(A-1)$-hole state, is expressed as follows:

$$
\begin{aligned}
T_{h}^{\gamma^{*} A} \equiv F_{(A-1)-h o l e, 0}=\int d^{3} r_{1} d^{3}\left\{r_{k}\right\} & \Psi_{0}^{A}\left(r_{1},\left\{r_{k}\right\}\right) \cdot \Psi_{h^{-1}}^{A-1^{+}}\left(\left\{r_{k}\right\}\right) \cdot T^{e m}\left(Q^{2}\right) e^{i \vec{q} \vec{r}_{1}} \\
& \times e^{-i \vec{p}_{i} \vec{r}_{1}} \prod_{i=2}^{A}\left[1-\Gamma^{N}\left(b_{1}-b_{i}\right) \cdot \Theta\left(z_{i}-z_{1}\right)\right] .
\end{aligned}
$$

Here $\Psi_{h^{-1}}^{A-1}\left(\left\{r_{k}\right\}\right)$ - is the wave function of $A-1$-hole state of residual nucleus and $\vec{p}_{i}=\vec{p}_{f}-\vec{q}$. To simplify the formulae, we shall always denote the coordinate of the knocked-out nucleon as $r_{1}$ and will omit the sum over various nucleons $j$. Here $\vec{p}_{f}$ is the momentum of the knocked out nucleon. The rescatterings of the knocked out nucleon off the individual nucleons of the residual nucleus are described in eq. (2) by the product of functions $\Gamma^{N}$. The profile function $\Gamma^{N}(b)$ is expressed through the $N N$ amplitude $\left(f^{N N}\right)$ as:

$$
\Gamma^{N}(b)=\frac{1}{2 \pi i k} \int \exp \left(i \vec{k}_{t} \vec{b}\right) \cdot f^{N N}\left(\vec{k}_{t}\right) d^{2} k_{t}
$$

where for $f^{N N}$ we use the normalization $\operatorname{Im} f^{N N}=\frac{k}{4 \pi} \sigma_{t o t} e^{\frac{b}{2} t}$.

To calculate the amplitude $T_{h}^{\gamma^{*} A}$ given by eq.(Z) it is convenient to approximate the ground state wave function according to Ref. [20] as a product of the Slater determinant, representing the uncorrelated ground state wave functions $\psi_{n}\left(r_{i}\right)$ and Jastrow-type correlated basis function $C_{h}\left(r_{i}, r_{k}\right)$ :

$$
\begin{aligned}
\Psi_{0}^{A}\left(r_{1}, \ldots, r_{A}\right) & =N(A !)^{-\frac{1}{2}} \operatorname{det}\left|\psi_{n}\left(r_{i}\right)\right| \prod_{k>i=1}^{A}\left(1+C_{h}\left(r_{i}, r_{k}\right)\right) \\
& =N \sum_{h} \omega_{h} \phi_{h}\left(r_{1}\right) \prod_{k>1}\left(1+C_{h}\left(r_{1}, r_{k}\right)\right) \Psi_{h^{-1}}^{(A-1)}\left(\left\{r_{k}\right\}\right)
\end{aligned}
$$

Here $w_{h}^{2}$ is the occupation probability for the nucleon orbital $h$. In the last part of eq. (田) we modify the correlated basis representation by introducing the single nucleon wave functions - $\phi_{h}\left(\vec{r}_{i}\right)$, which represent the overlap integral between exact $A$-body ground state wave function $-\Psi_{0}^{A}$ and $A-1$-body wave functions of the residual nucleus- $\Psi_{h^{-1}}^{(A-1)}\left(\left\{r_{k}\right\}\right)$. Single nucleon wave functions are normalized as follows:

$$
\int\left|\phi_{h}(r)\right|^{2} d^{3} r=1
$$

and 18,26 


$$
\rho(r)=\sum_{h} \omega_{h}^{2}\left|\phi_{h}(r)\right|^{2}
$$

where $\rho(r)$ is the single nucleon density function defined according to eqs.(38), (43), (44), which can be taken either from nuclear many-body calculations or from experimental data. Terms $C_{h}\left(r_{1}, r_{k}\right)$ in eq.(四) parameterize ground state pair nucleon correlations between detected nucleon 1 and undetected nucleons $-k$. The factor $N \approx 1+\mathcal{O}\left(l_{c}^{3} / R_{A}^{3}\right)$, accounts for the proper normalization, where $l_{c}$ - characterize the correlation lengths between nucleons [7] and $R_{A}$ is the nuclear radius (the normalization factor $N$ is further discussed in the Appendix). In the case when three-nucleon correlations in a nucleus can be neglected $N \approx 1$.

Correlations enter eq.(4) in two ways, through the correlation of the struck nucleon with nearby nucleons (via $C_{h}\left(r_{1}, r_{k}\right)$ functions) and through the correlations between "spectator" nucleons which are contained in the function $\Psi_{h^{-1}}^{A-1}$. Substituting, in eq.(2), the nuclear ground state wave function by eq.(四) we obtain:

$$
\begin{aligned}
T_{h}= & \sum_{h^{\prime}} \int d^{3} r_{1} d^{3}\left\{r_{k}\right\} \omega_{h^{\prime}} \phi_{h^{\prime}}\left(r_{1}\right) \prod_{k=2}^{A}\left(1+C_{h^{\prime}}\left(r_{1}, r_{k}\right)\right) \Psi_{h^{\prime-1}}^{(A-1)}\left(\left\{r_{k}\right\}\right) \cdot T^{e m}\left(Q^{2}\right) \cdot e^{-i \vec{p}_{i} \vec{r}_{1}} \\
& \times \prod_{i=2}^{A}\left[1-\Gamma^{N}\left(b_{1}-b_{i}\right) \cdot \Theta\left(z_{i}-z_{1}\right)\right] \Psi_{h^{-1}}^{(A-1)^{+}}\left(\left\{r_{k}\right\}\right) \\
= & \sum_{h^{\prime}} \int d^{3} r_{1} d^{3}\left\{r_{k}\right\} \omega_{h^{\prime}} \phi_{h^{\prime}}\left(r_{1}\right) \cdot T^{e m}\left(Q^{2}\right) \cdot e^{-i \vec{p}_{i} \vec{r}_{1}} \prod_{i=2}^{A}\left[1-\Gamma^{N}\left(b_{1}-b_{i}\right) \cdot \Theta\left(z_{i}-z_{1}\right)\right] \\
& \times\left[\prod_{k=2}^{A}\left(1+C_{h^{\prime}}\left(r_{1}, r_{k}\right)\right)\right] \cdot \rho_{h^{\prime}, h}^{A-1}\left(\left\{r_{k}\right\}\right) .
\end{aligned}
$$

To simplify formulae, we introduce the $A-1$-body density matrix as:

$$
\rho_{h^{\prime} h}^{A-1}\left(\left\{r_{k}\right\}\right) \equiv \Psi_{h^{\prime-1}}^{(A-1)}\left(\left\{r_{k}\right\}\right) \times \Psi_{h^{-1}}^{(A-1)^{+}}\left(\left\{r_{k}\right\}\right)
$$

which satisfy (as a consequence of the orthogonality condition described in Appendix) the following sum rules:

$$
\int \rho_{h^{\prime} h}^{A-1}\left(\left\{r_{k}\right\}\right) d^{3}\left\{r_{k}\right\}=\delta_{h^{\prime}, h},
$$

and

$$
\rho^{A-1}\left(\left\{r_{k}\right\}\right)=\sum_{h} \omega_{h}^{2}\left|\rho_{h, h}^{A-1}\left(\left\{r_{k}\right\}\right)\right|^{2},
$$


where $\rho^{A-1}\left(\left\{r_{k}\right\}\right)$ is the conventional $A-1$-body density function.

In eq.(7) hard electromagnetic and soft hadron-nucleus scattering are separated since we ignore the off-energy-shell effects in $T^{e . m \cdot}\left(Q^{2}\right)$. To calculate nuclear effects we may use the methods developed for the calculation of hadron-nucleus scattering [6] provided the $(A-1)$ nucleon density matrix (eq.(B) ) of residual nucleus is known. However, this matrix is practically unknown now. So to evaluate transitions to certain nuclear levels, we will neglect the contribution of nondiagonal $h \rightarrow h^{\prime}$ transitions basing on the following reasons: i) our interest is in the processes where fixed missing energy is small $(\lesssim 50 \mathrm{MeV})$ and we consider nuclei which have clear resolved shell structure (e.g. ${ }^{12} C$ ); ii) the nondiagonal transitions from large $E_{m}$-states to small $E_{m}$-state are strongly suppressed since FSI leads to further increase of the overall missing energy; iii) overlap integrals for the transition from small $E_{m^{-}}$ states to large $E_{m}$-state are suppressed too [18,26]; iv) the ability to fix missing momenta independently of the missing energy in the considered kinematics, allow to suppress further the nondiagonal transitions, ( for example, in the case of $p_{i} \approx 0$ the contributions from $l \neq 0$ orbits are suppressed as compared to the $l=0$ one); v) nondiagonal $h \rightarrow h^{\prime}$ transitions are a correction to the contribution of nucleon correlations into nuclear transparency. However we find below that effect of correlations in the processes we consider is smal. Neglecting the nondiagonal transitions between the residual nuclear states, we expand the $(A-1)$ body density function for a particular hole $-h$, similar to Refs. [7.8] through the (two, three, etc.)-body correlation functions:

$$
\rho_{h, h}^{A-1}\left(\left\{r_{k}\right\}\right)=\rho\left(r_{2}\right) \times \ldots \rho\left(r_{A}\right)+\sum_{i, j} g_{h}\left(r_{i}, r_{j}\right) \cdot \rho\left(r_{2}\right) \times \ldots \rho\left(r_{i}\right) \times . . \rho\left(r_{j}\right) . . \rho\left(r_{A}\right) \quad+\ldots
$$

where $\rho\left(r_{i}\right)$ is the above defined nucleon single density function. In the approximation when only pair nucleon correlations are kept, it follows from eqs.(9) and (10) (cf. Appendix) that:

$$
\int g_{h}\left(r_{i}, r_{j}\right) \rho\left(r_{i}\right) \rho\left(r_{j}\right) d^{3} r_{i} d^{3} r_{j}=0
$$

Using this decomposition of $(A-1)$-body density matrix (eq.(11)), we obtain for FSI practically the same functional form as that obtained in [7,8] for $h A$ scattering within the Glauber 
approximation, when two body correlations are taken into account. The major difference from [7,8] is the different geometry of $e A$ collisions as compared to $h A$ scattering. This difference is accounted for in eq.(可) in the limits of integration over the coordinate $z_{1}$ of the knocked out nucleon. Taking into account the normalization conditions for the correlation function $C\left(r_{i}, r_{j}\right)$ (see Appendix) and $g_{h}\left(r_{i}, r_{j}\right)$ (eq.(12) ) we obtain for the amplitude $T_{h}$ the expression similar to that in Ref. [7]:

$$
\begin{aligned}
T_{h}^{\gamma^{*} A} & =\int d^{3} r_{1} \cdot \omega_{h} \phi_{h}\left(r_{1}\right) \cdot T^{e m}\left(Q^{2}\right) \cdot e^{-i \vec{p}_{i} \vec{r}_{1}} \\
& \times\left[1-\int_{z_{1}} d^{3} r\left(1+C_{h}\left(r_{1}, r\right)\right) \rho(r) \Gamma\left(b_{1}-b\right)\right]^{(A-1)} \cdot \mathcal{P}_{A-1}\left(\left(1+C_{h}\right), \Gamma\right),
\end{aligned}
$$

where the factor $\mathcal{P}_{A-1}\left(\left(1+C_{h}\right), \Gamma\right)$, characterizes influence of correlations between undetected nucleons on FSI of knocked-out nucleon [7]:

$$
\begin{aligned}
& \mathcal{P}_{A-1}\left(\left(1+C_{h}\right), \Gamma\right)=\sum_{m=0}^{\frac{A-1}{2} o r \frac{A-2}{2}} \frac{(A-1) !}{(A-1-2 m) ! m !} \\
& \times\left[\frac{\frac{1}{2} \int_{z_{2,3}>z_{1}} d^{3} r_{2} d^{3} r_{3}\left(1+C_{h}\left(r_{1}, r\right)\right) g_{h}\left(r_{2}, r_{3}\right) \rho\left(r_{2}\right) \rho\left(r_{3}\right) \Gamma\left(b_{1}-b_{2}\right) \Gamma\left(b_{1}-b_{3}\right)}{\left(1-\int_{z>z_{1}} d^{3} r\left(1+C_{h}\left(r_{1}, r\right)\right) \rho(r) \Gamma\left(b_{1}-b\right)\right)^{2}}\right]^{m} .
\end{aligned}
$$

Eqs.(13) and (14) show that correlations between undetected nucleons enter similarly to $h A$ scattering [7],8], while correlations between the knocked-out nucleon and undetected nucleons enter via the rescaling of the single nucleon density function by the factor $\left(1+C_{h}\left(r_{1}, r\right)\right)$.

At large $A$, eqs.(13), (14) can be considerably simplified by keeping in the factor [. . $]^{m}$ in eq. (14) only the terms which grow with $A$. The formulae obtained for large $A$ resemble the optical limit of Glauber approximation formulae:

$$
T_{h}^{\gamma^{*} A}=\int d^{3} r_{1} \cdot \omega_{h} \phi_{h}\left(r_{1}\right) \cdot T^{e m}\left(Q^{2}\right) \cdot e^{-i \vec{p}_{i} \vec{r}_{1}} e^{-\int \Gamma\left(b_{1}-b\right) \tilde{n}(r) d^{3} r},
$$

where $\tilde{n}(r) \equiv(A-1) \cdot \tilde{\rho}(r)$. The modified nuclear density is:

$$
\begin{aligned}
\tilde{\rho}(z, b) & =\left[1+C_{h}\left(r_{1}, r\right)\right]\left(1-\frac{A-1}{2} \int_{z_{1}} \Gamma\left(b_{1}-b^{\prime}\right) g_{h}\left(r, r^{\prime}\right) 1 \rho\left(r^{\prime}\right) d^{3} r^{\prime}\right) \rho(r) \\
& \approx \rho(r)\left[1+C_{h}\left(r_{1}, r\right)-\frac{A-1}{2} \int_{z_{1}} \Gamma\left(b_{1}-b^{\prime}\right) g_{h}\left(r, r^{\prime}\right) \rho\left(r^{\prime}\right) d^{3} r^{\prime}\right],
\end{aligned}
$$


where, at the last step, we neglect the term proportional to the square of correlations, since its contribution is comparable to higher order correlations which were neglected earlier. In practice eq.(15) is applicable starting from $A \geq 4$, with accuracy comparable to the accuracy of exponentiation of $(A-1)$-power function in eq. 13$)$, estimated as $\sim \mathcal{O}\left(\frac{1}{2 A}\left[\int_{z>z_{1}} \Gamma\left(b_{1}-\right.\right.\right.$ b) $\left.\left.\tilde{n} d^{3} r\right]^{2}\right)$.

In the limit when correlations are neglected the derived equations coincide with the formulae of the Glauber approximation in the independent particle approximation for the nuclear wave function. When FSI is neglected, the derived formulae lead to plane wave impulse approximations within the generalized shell model, where correlations in the ground state wave functions are taken into account (see e.g. [18,21,22]).

The interesting feature of eqs.(15), (16) is that account of nucleon correlations influence the final state interaction in two opposite ways. Due to the second term in eq.(16), correlations lead to decrease of the effective nuclear densities $\left(C_{h}\left(r, r^{\prime}\right)<0\right)$ and therefore to increase of the transparency of nuclear matter for the knocked-out nucleon. This effect reflects the presence of a hole around the scattered nucleon in the ground state wave functiosendn. This effect has been previously mentioned in Ref. [19 and analyzed at length in [12], within the optical approximation and for cross sections integrated over missing momentum and energy.

However, the contribution of correlations in the third term of eq.(16) leads to increase of the effective nuclear density and as a result, nuclear matter become more opaque for the knocked-out nucleon. Same effect was found in Refs. [6 8] for high energy hadron-nucleus scattering. Similar effect was discussed also in Refs. [14,16, 17] for the cross section of $\left(e, e^{\prime} p\right)$ reaction summed over the final states of residual nucleus and integrated over the proton momentum. In this case this contribution into the overall correlation effect is practically negligible [16,17.

To calculate the cross section of the semiexclusive $\left(e, e^{\prime} N\right)$ reaction we use the distorted wave impulse approximation (DWIA) where the cross section can be represented as follows: 


$$
\frac{d^{6} \sigma}{d \epsilon_{2} d \Omega_{2} d^{3} \vec{p}_{f}}=\sigma_{e N} \cdot S_{A}\left(\vec{p}_{i}, E m, \vec{p}_{f}\right),
$$

where $\sigma_{e N}$ is proportional to the cross section of electron scattering off a bound nucleon. We restrict ourselves to the case of reactions where nucleon Fermi motion is small (i.e., small missing momenta). For large missing momenta, more accurate treatment of multistep processes and relativistic effects is necessary.

If the specific shell is fixed the DWIA spectral function can be written as [18]:

$$
S_{A}\left(\vec{p}_{i}, E_{m}, \vec{p}_{f}\right)=n_{h}\left(E_{m}\right) \cdot\left|\Phi_{h}\left(\vec{p}_{i}, \vec{p}_{f}\right)\right|^{2},
$$

where $n_{h}\left(E_{m}\right)$ characterizes the strength of the shell and proportional to the shell occupation probabilities $\sim \omega_{h}^{2}$. $\Phi_{h}\left(\vec{p}_{i}, \vec{p}_{f}\right)$ is the distorted momentum distribution of the nucleons for $h$-shell. Using eq.(15) we obtain for $\left|\Phi_{h}\left(\vec{p}_{i}, \vec{p}_{f}\right)\right|^{2}$ :

$$
\left|\Phi_{h}\left(\vec{p}_{i}, \vec{p}_{f}\right)\right|^{2}=\left|\int d^{3} r_{1} \Psi_{h}\left(r_{1}\right) e^{-i \vec{p}_{i} \vec{r}_{1}} \times\left[e^{-\int \sum\left(b_{1}-b\right) \tilde{n}(r) d^{3} r}\right]\right|^{2} .
$$

\section{NUCLEAR TRANSPARENCY}

We use eq.(15) for the scattering amplitude and eq.(19), for the distorted momentum distributions to calculate $\left(e, e^{\prime} N\right)$ scattering on nuclei.

In this section we will consider the effects which are due to the final state interaction of knocked - out nucleons. The convenient quantity to characterize the FSI (see e.g. Refs. [1, 12, 11, 32]) is the ratio of the measured cross section of the $\left(e, e^{\prime} N\right)$ reactions and the cross section calculated within the plane wave impulse approximation (PWIA). In the case of complete nuclear transparency this ratio will equal unity. The corresponding theoretical quantity is the ratio of the cross sections calculated with and without FSI.

In the theoretical analysis a convenient quantity is the transparency corresponding to a transition when the particular nuclear shell is fixed (no summation over the final states of residual nucleus): 


$$
T_{r}^{h} \equiv\left(\frac{\sigma^{E X P}}{\sigma^{P W I A}}\right)_{h}=\frac{\left|\Phi_{h}^{D W I A}\left(p_{i}, p_{f}\right)\right|^{2}}{\left|\Phi_{h}^{P W I A}\left(p_{i}\right)\right|^{2}}
$$

where $\left|\Phi_{h}^{D W I A}\left(p_{i}, p_{f}\right)\right|^{2}$ is given by eq.(19).

\section{A. Qualitative estimates}

To visualize the role of nucleon-nucleon correlations it is worth considering first a highly simplified model of uniform nuclear density. We will also treat nucleons as point-like and furthermore approximate correlation functions as $\Theta$-functions:

$$
C_{h}(x) \approx g(x) \approx-\Theta\left(l_{c}-x\right)
$$

where $l_{c}$ is the correlation length defined as [0]:

$$
l_{c}=-\int_{0}^{\infty} g(r) d r
$$

Here $g(r)$ is the correlation function calculated within the realistic theory of nucleus. We will omit $T^{e m}\left(Q^{2}\right)=1$ in the next following analysis since electromagnetic form factors of a nucleon are canceled in eq.(20) for nuclear transparency provided the off-shell effects are neglected.

The profile function of point-like nucleon is (cf eq.(3)):

$$
\Gamma\left(b_{1}-b_{2}\right)=\frac{\sigma_{t o t}}{2} \cdot \delta^{2}\left(b_{1}-b_{2}\right) \text {. }
$$

Using the above approximations and assuming that,

$$
\int_{z_{1}} g\left(z-z_{1}\right) \rho\left(z, b_{1}\right) d z \approx-2 l_{c} \rho\left(z_{1}, b_{1}\right)
$$

we obtain for the exponent in eq.(15) :

$$
-\int_{z_{1}} \Gamma\left(b_{1}-b\right) \tilde{n}(r) d^{3} r=-\frac{\sqrt{R^{2}-b_{1}^{2}}-z}{2 \lambda}-\frac{l_{c}}{\lambda}\left[\frac{\sqrt{R^{2}-b_{1}^{2}}-z}{4 \lambda}-1\right],
$$

where $R$-is the nuclear radius and $\lambda=\frac{1}{\sigma \rho_{0}}$ is the mean free path, and $\rho_{0}$ is the uniform density. Approximating the one-body wave function as uniform: 


$$
\phi_{h}=\frac{\Theta(|R-r|)}{\left(\frac{4 \pi}{3} R^{3}\right)^{\frac{1}{2}}}
$$

we calculate the amplitude $F^{f 0}$ with coherent FSI in the case of $\vec{p}_{i}=\vec{p}_{f}-\vec{q}=0$ for transition to the (A-1) nucleon system:

$$
F^{c o h}=\frac{e^{\frac{L}{\Lambda}}\left(\frac{4 \pi}{3} R\right)^{\frac{1}{2}}}{\frac{1}{\Lambda}+\frac{L}{2 \Lambda^{2}}} \cdot \frac{3}{2}\left[1+\frac{2\left(e^{-\left(\frac{1}{\Lambda}+\frac{L}{2 \Lambda^{2}}\right)}\left(1+\frac{1}{\Lambda}+\frac{L}{2 \Lambda^{2}}\right)-1\right)}{\left(\frac{1}{\Lambda}+\frac{L}{2 \Lambda^{2}}\right)^{2}}\right]
$$

and for the transparency defined as in eq.(20) (for certainty we consider the case $\mathrm{k}=0$ ):

$$
T_{r}^{c o h}=\frac{\left|F^{c o h}\right|^{2}}{\left|\phi_{h}(k=0)\right|^{2}}=\frac{e^{\frac{2 L}{\Lambda}}}{\left(\frac{1}{\Lambda}+\frac{L}{2 \Lambda^{2}}\right)^{2}} \cdot \frac{9}{4}\left[1+\frac{2\left(e^{-\left(\frac{1}{\Lambda}+\frac{L}{2 \Lambda^{2}}\right)}\left(1+\frac{1}{\Lambda}+\frac{L}{2 \Lambda^{2}}\right)-1\right)}{\left(\frac{1}{\Lambda}+\frac{L}{2 \Lambda^{2}}\right)^{2}}\right]^{2},
$$

where $L \equiv \frac{l_{c}}{R}$ and $\Lambda \equiv \frac{\lambda}{R}$.

To visualize the effects of correlations in eqs.(28) it is convenient to normalize transparency to the corresponding transparencies within the uncorrelated Glauber approximation and to consider the case when $A$ is sufficiently large and $\frac{1}{\Lambda} \gg 1, \frac{L}{\Lambda}=\frac{l_{c}}{\lambda} \ll 1$.

We obtain for this ratio:

$$
\left(\frac{T_{r}}{T_{r}^{\text {noncorr }}}\right)^{\text {coh }} \approx \frac{e^{\frac{2 L}{\Lambda}}}{\left(1+\frac{L}{2 \Lambda}\right)^{2}} \approx\left(1+\frac{L}{\Lambda}\right) .
$$

Eq.(29) clearly demonstrates that correlation between detected nucleon and non detected nucleons (numerator in eq.(29)) and correlations between undetected nucleons (denominator in eq.(29) ) enter differently into nuclear transparency and the first effect dominates.

The next important feature of coherent rescatterings for transitions to a ground state is the strongly different $A$ dependence of nuclear transparency compared to the case of incoherent final state interactions $\sim A^{-\frac{1}{3}}$ ( see e.g. [32]). From eq.(28) we obtain:

$$
T_{r}^{c o h} \sim \Lambda^{2} \sim R^{-2} \sim A^{-\frac{2}{3}}
$$

This considerably oversimplified model of the nucleus demonstrates the qualitative difference between coherent and incoherent final state interactions. The obtained formulae show that fixing the final states of residual nucleus is more promising for searching color transparency effects in nuclei since CT effects are larger in this case. 


\section{B. Quantitative calculations}

We present here numerical results for the case of $\left(e . e^{\prime} p\right)$ scattering off ${ }^{12} C$ and use the kinematics where momentum of knocked-out proton is equal to the transferred momentum: $\vec{p}_{f}=\vec{q}$. The distinguishable shell structure of ${ }^{12} C$ allows us to outline the effects of shell structure on the nuclear transparency. In calculations of the ${ }^{12} C$ ground state wave functions we use the Skyrme - Hartree - Fock model with correlated interaction [26].

To describe the correlation properties of the nuclear ground state and final $(A-1)$-hole state, we assume that NN pair correlations are state independent. This assumption is inferred from both theoretical and experimental observations (see e.g. [10,27,28]) indicating that the nuclear high momentum components (controlled mainly by short range NN correlations) are practically the same for all nuclei. This approximation is not reliable for the long range correlations, where strong density dependence (observed in Ref. [24]) should be taken into account. For our calculations we use the correlation function $g(r)$ from the calculation of [24,25 for standard nuclear density $\left(=0.16 \mathrm{fm}^{-3}\right)$. .f The accuracy of such approximation depends on the overall size of correlation effects and use of the exact density-dependent correlation function would clearly improve the present calculations.

The profile functions in eq.(3) have been calculated using the relation

$$
\left|f\left(k_{t}\right)\right|^{2}=\frac{k^{2}}{\pi} \frac{d \sigma}{d t}
$$

For $\frac{d \sigma}{d t}$ we use the phenomenological parameterization:

$$
\frac{d \sigma}{d t}=\frac{\sigma_{t o t}^{2}}{16 \pi}\left(1+\alpha^{2}\right) \exp (b t)
$$

where $\alpha=\operatorname{Ref} / \operatorname{Imf}$ and all parameters are taken from [29 31].

In fig. 1 we present the results of calculations of the $Q^{2}$ dependence of the nuclear transparency based on eq.(20) for the proton knocked out from the $s$ shell. We observe that two

\footnotetext{
* The function $g(r)$ used in present work is related to the correlation function $g_{0}(r)$ obtained in 24,25] as $g(r)=\frac{A}{A-1} g_{0}(r)-1$.
} 
opposite effects of nucleon correlations reduce the overall effect of correlations to the level of few percent.

To see the interplay of the above discussed effects with the anticipated effects of color transparency (CT) we use the quantum diffusion model (QDM) [32 to account for the reduction of FSI for knocked-out proton due to CT effects. For this purpose we introduce the modified profile function in eq.(33) with the modified $N N$ scattering amplitude [33]:

$$
f^{N N}\left(k_{t}, Q^{2}, l\right) \approx i \frac{k}{4 \pi} \sigma_{t o t}\left(l, Q^{2}\right) \cdot e^{\frac{b}{2} t} \cdot \frac{G_{N}\left(t \cdot \sigma_{t o t}\left(l, Q^{2}\right) / \sigma_{t o t}\right)}{G_{N}(t)},
$$

where $b$ is the slope of elastic $N N$-cross section and $G_{N}(t)\left(\approx(1-t / 0.71)^{2}\right)$ is the Sachs form factor. The last factor in eq. (33) accounts for the difference between form-factors for point-like and average configurations, which is estimated based on the observation that the $t$ dependence of $d \sigma^{h+N \rightarrow h+N} / d t \sim G_{h}^{2}(t) \cdot G_{N}^{2}(t)$. The effective $N N$ total cross section we calculate using the (QDM) predictions [32]:

$$
\sigma_{\text {tot }}\left(l, Q^{2}\right)=\sigma_{t o t}\left\{\left(\frac{l}{l_{h}}+\frac{<r_{t}\left(Q^{2}\right)^{2}>}{<r_{t}^{2}>}\left(1-\frac{l}{l_{h}}\right)\right) \Theta\left(l_{h}-l\right)+\Theta\left(l-l_{h}\right)\right\},
$$

where $l_{h}=2 p_{f} / \Delta M^{2}$, with $\Delta M^{2}=0.7 \mathrm{GeV}^{2} \cdot \frac{\left\langle r_{t}\left(Q^{2}\right)^{2}>\right.}{<r_{t}^{2}>} \approx \frac{1 G e V^{2}}{Q^{2}}$ - is the average transverse size squared of the configuration produced in the interaction point.

In fig.2 we present the $Q^{2}$ dependence of the color transparency effect in the kinematics of fig.1 (curves labeled "s-shell"). Fig.2 shows that the correlation effect becomes smaller at high $Q^{2}$ since according to Eqs.(15)-(16) the reduced value of the cross section of $N N$ scattering reduces sensitivity to the changes of density function $\rho \rightarrow \tilde{\rho}$. However correlations slightly reduce the onset of CT effect since the nucleus becomes more transparent.

Another important effect, in the kinematics of coherent FSI, is different manifestation of color transparency for fixed $s$ and $p$ shells. It follows from fig.3 that in the case of the proton knock-out from $p$ and $s$ shells in the kinematics where $\vec{p}_{i}=\vec{p}_{f}-\vec{q} \approx 0$ the decrease of FSI leads to opposite effects on the cross section for $\left(e, e^{\prime} p\right)$ reaction for s- and p-shells. In fig.4 the expected CT effects calculated using eqs.(33,34) normalized to the corresponding transparencies within the Glauber approximation. 


\section{CONCLUSIONS}

We investigated the final state interaction of knocked out nucleons in high $Q^{2}\left(e, e^{\prime} N\right)$ processes within the Glauber approximation, taking into account the nucleon correlations in a consistent way in the nuclear ground state and fixed $A-1$ final state wave function. The main conclusion is that nucleon correlations affect the nuclear transparency in two different ways: correlations among undetected nucleons make a nucleus less transparent, while the correlations among the detected nucleon and undetected nucleons make the nucleus more transparent.

The consideration of nuclear shell structure shows that effects of correlations are on the level of few percent for coherent final state interaction.

By including color coherent effects we elaborate the observation of Ref. [11] that color transparency have different implications for the excitation of different nuclear shells. We

conclude that $\left(e, e^{\prime} p\right)$ reactions are more sensitive to the color coherent effects provided the particular nuclear shell is fixed.

\section{ACKNOWLEDGMENTS}

This work was supported in part by the U.S. Department of Energy under grants DEFG02-93ER40771 and DE-FG02-94ER40823, and Israel- USA Binational Science Foundation Grant No. 9200126. EJM is grateful for support from the Alexander von Humboldt Stiftung.

\section{APPENDIX}

To construct the wave function of the nuclear ground state via single-nucleon wave function - $\phi_{h}\left(r_{1}\right)$ and $(A-1)-$ hole wave function - $\Psi_{h^{-1}}^{(A-1)}\left(\left\{r_{k}\right\}\right)$ we introduce the $h$-state dependent correlation functions between nucleon at $r_{1}$ and $A-1$ nucleons belonging to the rest of the nucleus: 


$$
\Psi_{0}^{A}\left(r_{1}, \ldots, r_{A}\right)=N \sum_{h} \omega_{h} \phi_{h}\left(r_{1}\right) \prod_{k>1}\left(1+C_{h}\left(r_{1}, r_{k}\right)\right) \Psi_{h^{-1}}^{(A-1)}\left(\left\{r_{k}\right\}\right)
$$

where $\left\{r_{k}\right\} \equiv r_{2}, \ldots r_{A}$.

$$
\int\left|\Psi_{0}^{A}\left(\left\{r_{k}\right\}\right)\right|^{2} d^{3}\left\{r_{k}\right\}=1
$$

We choose normalization of $\Psi_{h^{-1}}^{(A-1)}$ as

$$
\int \Psi_{h^{-1}}^{(A-1)} \Psi_{h^{\prime-1}}^{(A-1)} d^{3}\left\{r_{k}\right\}=\delta_{h h^{\prime}}
$$

The overall normalization factor $N \approx 1$ provided three(or more)-nucleon correlations are neglected. To reproduce the formulae of the shell model we choose $\rho\left(r_{1}\right)$ as

$$
\rho\left(r_{1}\right)=\int \rho_{A}\left(r_{1},\left\{r_{k}\right\}\right) d^{3}\left\{r_{k}\right\}=\int\left|\Psi_{0}^{A}\left(r_{1},\left\{r_{k}\right\}\right)\right|^{2} d^{3}\left\{r_{k}\right\}=\sum_{h} \omega_{h}^{2} \phi_{h}^{2}\left(r_{1}\right)
$$

where $\rho\left(r_{1}\right)$-is the single nucleon density function.

As a consequence of the orthogonality of $h$-state wave functions - eq.(36) and eq.(38) the correlation functions $C_{h}\left(r_{1}, r_{k}\right)$ should obey the following relations:

$$
\int\left[\prod_{k>1}\left(1+C_{h}\left(r_{1}, r_{k}\right)\right) \prod_{k>1}\left(1+C_{h^{\prime}}^{+}\left(r_{1}, r_{k}\right)\right)-1\right] \Psi_{h^{-1}}^{(A-1)}\left(\left\{r_{k}\right\}\right) \Psi_{h^{\prime-1}}^{(A-1)^{+}}\left(\left\{r_{k}\right\}\right) d^{3}\left\{r_{k}\right\}=0 .
$$

if three ...nucleon correlations are neglected. For $h=h^{\prime}$, within the accuracy $\mathcal{O}\left(C_{h}^{2}\right)$ we obtain:

$$
\int C_{h}\left(r_{k}, r_{k}\right)\left|\Psi_{h^{-1}}^{(A-1)}\left(\left\{r_{k}\right\}\right)\right|^{2}=0
$$

For the practical aims we neglect the dependence of the $(A-1)$-nucleus wave function $\left|\Psi_{h^{-1}}^{(A-1)}\left(\left\{r_{k}\right\}\right)\right|^{2} \equiv \rho^{(A-1)}\left(\left\{r_{k}\right\}\right)$ and the correlation function $C_{h}\left(r_{1}, r_{k}\right) \equiv C\left(r_{1}, r_{k}\right)$ on the nucleon orbital h expressing them through the correlation function $g\left(r_{i}, r_{j}\right)$ defined as:

$$
\begin{gathered}
\int\left|\Psi_{0}^{A}\left(r_{1}, r_{2} . ., r_{A}\right)\right|^{2} d^{3} r_{1} d^{3} r_{i-1} d^{3} r_{i+1} d^{3} t_{j-1} d^{3} r_{j+1} d^{3} r_{A}=\rho_{2}\left(r_{i}, r_{j}\right)= \\
\left(\left(1+g\left(r_{i}, r_{j}\right)\right) \cdot \rho\left(r_{i}\right) \cdot \rho(j)\right.
\end{gathered}
$$

where two-nucleon density function $\rho_{2}\left(r_{i}, r_{j}\right)$ is the probability to find $(i, j)$ nucleons $(i, j)$ in nucleus simultaneously at the points $r_{i}$ and $r_{j} . \rho_{2}$ is normalized as: 


$$
\int \rho_{2}\left(r_{i}, r_{j}\right) d^{3} r_{i} d^{3} r_{j}=1
$$

The single nucleon density $\rho(r)$ is:

$$
\int \rho_{2}\left(r, r^{\prime}\right) d^{3} r^{\prime}=\rho(r)
$$

It is normalized as:

$$
\int \rho(r) d^{3} r=1
$$

Inserting eq.(41) to eq.(42) and using eq.(44) we obtain the following sum rule for the correlation function $g\left(r_{i}, r_{j}\right)$ :

$$
\int g\left(r_{i}, r_{j}\right) \rho\left(r_{i}\right) \rho\left(r_{j}\right) d^{3} r_{i} d^{3} r_{j}=0
$$

Inserting eq.(41) to eq.(43)we obtain also:

$$
\int g\left(r_{i}, r_{j}\right) \rho\left(r_{i}\right) d^{3} d r_{i}=\int g\left(r_{i}, r_{j}\right) \rho\left(r_{j}\right) d^{3} r_{j}=0
$$

Expanding the $A$ body density function through the above defined correlation function $g$ as:

$$
\rho^{(A)}\left(r_{1},\left\{r_{k}\right\}\right)=\rho\left(r_{1}\right) \prod_{k=2}^{A}\left(1+g\left(r_{1}, r_{k}\right)\right) \rho^{(A-1)}\left(\left\{r_{k}\right\}\right),
$$

and comparing it with $A$ body density function defined by square modulus of eq.(35) one obtain following relations between two correlation functions:

$$
\left|1+C\left(r_{1}, r_{k}\right)\right|^{2}=1+g\left(r_{1}, r_{k}\right)
$$

Obtained relation allows to estimate the accuracy of the approximation $N=1$. Using the eqs.(35) and (48) and the normalization condition for $\Psi_{0}^{A}\left(r_{1}, \ldots, r_{A}\right)$ we obtain:

$$
\begin{aligned}
1= & N^{2}\left[1+\sum_{i, j} \int g\left(r_{i}, r_{j}\right) \rho\left(r_{i}\right) \rho\left(r_{j}\right) d^{3} r_{i} d^{3} r_{j}\right. \\
& +\sum_{i \leftrightarrow j, k} \int g\left(r_{i}, r_{j}\right) g\left(r_{i}, r_{k}\right) \rho\left(r_{i}\right) \rho\left(r_{j}\right) \rho\left(r_{k}\right) d^{3} r_{i} d^{3} r_{j} d^{3} r_{k} \\
& +\sum_{i \neq j \neq k \neq m} \int g\left(r_{i}, r_{j}\right) g\left(r_{k}, r_{m}\right) \rho\left(r_{i}\right) \rho\left(r_{j}\right) \rho\left(r_{k}\right) \rho\left(r_{m}\right) d^{3} r_{i} d^{3} r_{j} d^{3} r_{k} d^{3} r_{m} \\
& \left.+\sum_{i \leftrightarrow j, k} \int g\left(r_{i}, r_{j}\right) g\left(r_{i}, r_{k}\right) g\left(r_{j}, r_{k}\right) \rho\left(r_{i}\right) \rho\left(r_{j}\right) \rho\left(r_{k}\right) d^{3} r_{i} d^{3} r_{j} d^{3} r_{k}+\ldots\right]
\end{aligned}
$$


Taking into account the sum rules for function $g\left(r_{i}, r_{j}\right)$ (eqs.45) and (46)) we find that first nonvanishing term in eq.(49) is proportional to $\sim g^{3}$. In the framework of the uniform density model of nucleus ( see section 4.1) we obtain that:

$$
N \approx 1+\mathcal{O}\left(\frac{l_{c}^{3}}{R_{A}^{3}}\right)
$$

where $R_{A}$ is the nuclear radius and $l_{c}$ - is the correlation length defined in eq.(22). Using the estimation $l_{c} \approx 0.74 \mathrm{fm}$ Ref. [7] for nuclei with $A \geq 12$ the accuracy of condition $N=1$ is better than $1-2 \%$. The effect of neglected three-nucleon correlations is expected to be on same level, since they proportional to $\sim l_{c}^{3}$. 


\section{REFERENCES}

[1] N. C. R. Makins et al., Phys. Rev. Lett. 72, 1986 (1994).

[2] A. Mueller, in Proceedings of the 17 Rencontre de Moriond edited by J. Tranh Thanh Van (Editions Frontieres, Gif-sur-Yvette, 1982), p.13.

[3] S. J. Brodsky, in Proceedings of the Thirteenth International Symposium on Multiparticle Dynamics, edited by E.W. Kittel, W. Metzger, and A. Stergiou (World Scientific, Singapore, 1982), p.964.

[4] L. L. Frankfurt, G. A. Miller, and M. I. Strikman, Comments on Particle and Nuclear Physics, v.21, 1 (1992); L. L. Frankfurt, G. A. Miller, and M.Strikman, Nucl. Phys. A555, 752 (1993).

[5] A. S. Carroll et al., BNL experiment 850.

[6] E. J. Moniz, G. D. Nixon, Phys. Lett. 30B, 393 (1969); E. J. Moniz, G. D. Nixon and J. D. Walecka, in High-Energy Physics and Nuclear Structure, edited by S. Devons, ( Plenum Press - 1970) p.321.

[7] E. J. Moniz and G. D. Nixon, Annals of Physics 67, 58 (1971).

[8] D. R. Yennie in Hadronic Interactions of Electrons and Photons ed. J. Cummings, D. Osborn, 321 (1971).

[9] R. J. Glauber, Lectures in Theoretical Physics, Vol.1, eds. W. Brittain ans L. G. Dunham, New-York, 1959.

[10] L. L. Frankfurt and M. I. Strikman, Phys. Rep. 76, 215 (1981).

[11] L. L. Frankfurt, M. I. Strikman and M. Zhalov, Phys. Rev. C 50, 2189 (1994).

[12] O. Benhar et al., Phys. Rev. Lett. 69, 881 (1992).

[13] T.-S. H. Lee and G. A. Miller, Phys. Rev. C 45 ,1863 (1992). 
[14] N. N. Nikolaev, A. Szczurek, et al., Phys. Lett. B317, 287 (1993).

[15] A. Kohama, K. Yazaki, and R. Seki, Nucl.Phys. A551, 687 (1993).

[16] A. S. Rinat and M. F. Taragin, WIS-94/43, (1994).

[17] S. Frankel, W. Frati and N. R. Walet, UPR-498T2 1994.

[18] S. Frullani and J. Mougey, Advances in Nuclear Physics, 14, Plenum Press, New York, 1984.

[19] L. L. Frankfurt, M. I. Strikman, and M.Zhalov, Nucl. Phys. A515, 599 (1990).

[20] W. Weise, Nucl. Phys. A193, 625 (1972).

[21] A. E. L. Dieperink and T. de Forest, Jr., Ann. Rev. Nucl. Sci. 25, 1 (1975).

[22] G. van der Steenhoven, Ground-state correlations in ${ }^{12} \mathrm{C}$ and the mechanism of the $\left(e, e^{\prime} p\right)$ reaction, 1987.

[23] L. Frankfurt, E. Piasetsky, M. Sargsyan, and M. Strikman, Phys. Rev. C, 51, $N^{o} 2$ (1995).

[24] S. C. Pieper and V. R. Pandharipande, PHY-6866-TH-91.

[25] S. C. Pieper, Private Communications.

[26] P. G. Reinhard, The Skyrme - Hartree - Fock Model of the Nuclear Ground State, in Computational Nuclear Physics, v1, edt. K. Langake, J.A. Maruhn, S.E. Koonin, Springer-Verlag - 1991.

[27] W. Bertozzi, R. W. Lourie and E. J. Moniz, in Modern Topics in Electron Scattering Editors B. Frois and I. Sick, World Scientific - 1991, p.419.

[28] C. Marchand et al., Phys. Rev. Lett. 60, 1703 (1988).

[29] A. V. Dobrovolsky et al., Nucl.Phys. B214, 1, (1983). 
[30] B.H. Silverman et al., Nucl.Phys. A499, 763 (1989).

[31] Review of Particle Properties, Phys.Rev. D 45, 1 (1992).

[32] G.R. Farrar, L.L. Frankfurt, H. Liu, and M.I. Strikman, Phys. Rev. Lett. 61, 686 (1988).

[33] K. Sh. Egiyan, L. L. Frankfurt, W. R. Greenberg, G. A. Miller, M. M. Sargsyan, and M. I.Strikman, Nucl. Phys. A580, 365 (1994). 


\section{Figure Caption}

Figure 1. The $Q^{2}$ dependence of the nuclear transparency $T_{r}$ of ${ }^{12} C$, calculated according to eq. (20) for the reaction of proton knock-out from $s$-shell with $\vec{p}_{i}=0$ including the energy dependence of the $N N$ amplitudes. Dotted line - is the calculation without correlation effects, dashed line - with the effects of correlation between undetected nucleons, dash-dotted line - with the effects of correlation between knocked-out proton and undetected nucleons and solid line - with overall correlation effects.

Figure 2. The $Q^{2}$ dependence of color transparency effect defined by eqs.(20), corresponding to the kinematics of fig.1. Dashed line - without, solid line - with overall correlation effects.

Figure 3. The $Q^{2}$ dependence of color transparency effect in distorted momentum distribution of proton on $s$ and $p$ shells. Dashed line - without, solid line - with overall correlation effects.

Figure 4. The $Q^{2}$ dependence of color transparency effect normalised to the corresponding transparency within Glauber approximation. Curves labeled $s-$ shell, $p$-shell corresponded to the fixed shell scattering, with coherent FSI, from $s$-shell, $p$-shell. For all cases $\vec{p}_{i}=0$. Dashed line - is the calculation without correlation effects, solid line - with correlation effects. 
This figure "fig1-1.png" is available in "png" format from: http://arxiv.org/ps/nucl-th/9501019v1 


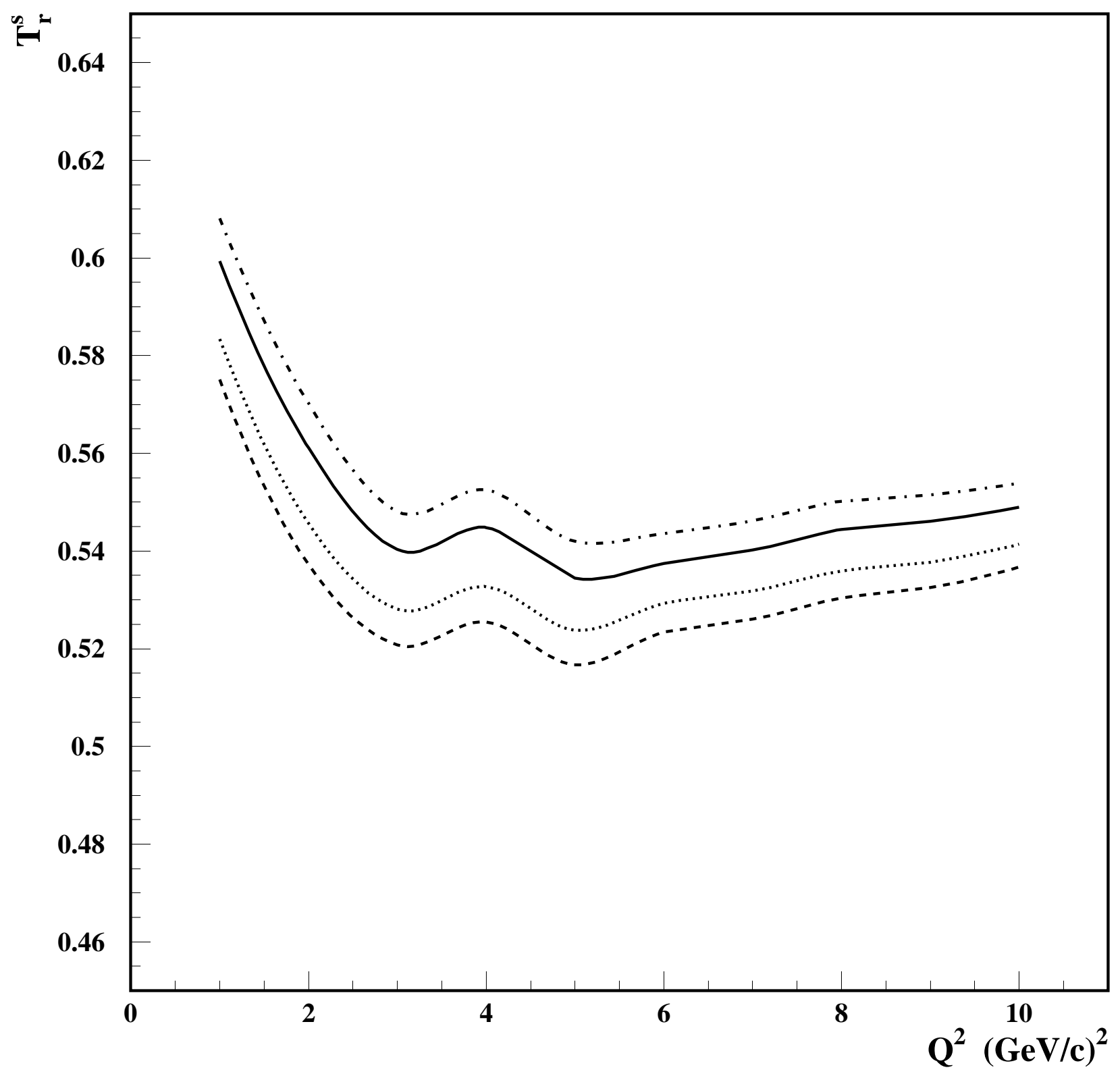

Fig.1 
This figure "fig1-2.png" is available in "png" format from: http://arxiv.org/ps/nucl-th/9501019v1 


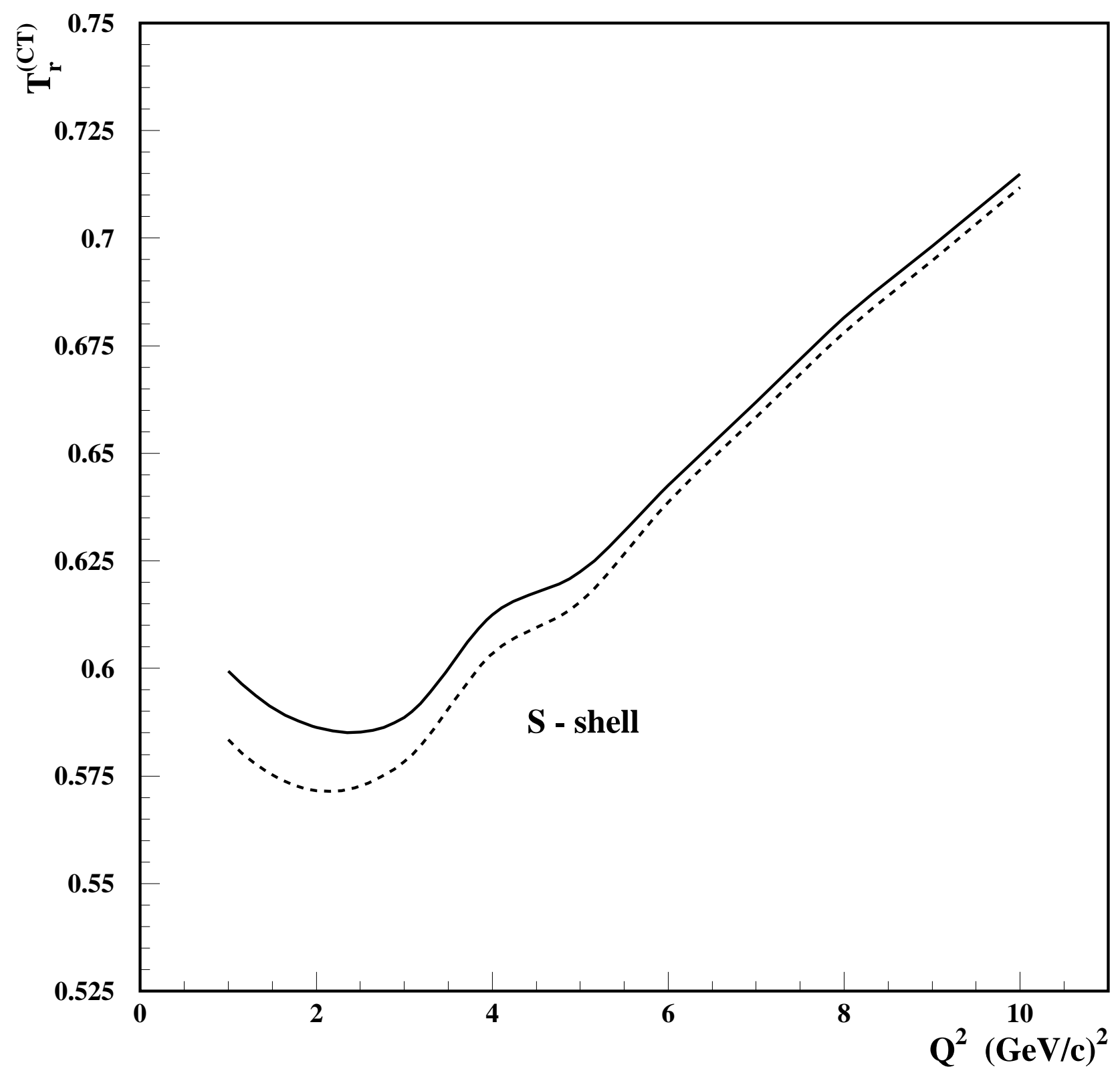

Fig.2 
This figure "fig1-3.png" is available in "png" format from: http://arxiv.org/ps/nucl-th/9501019v1 


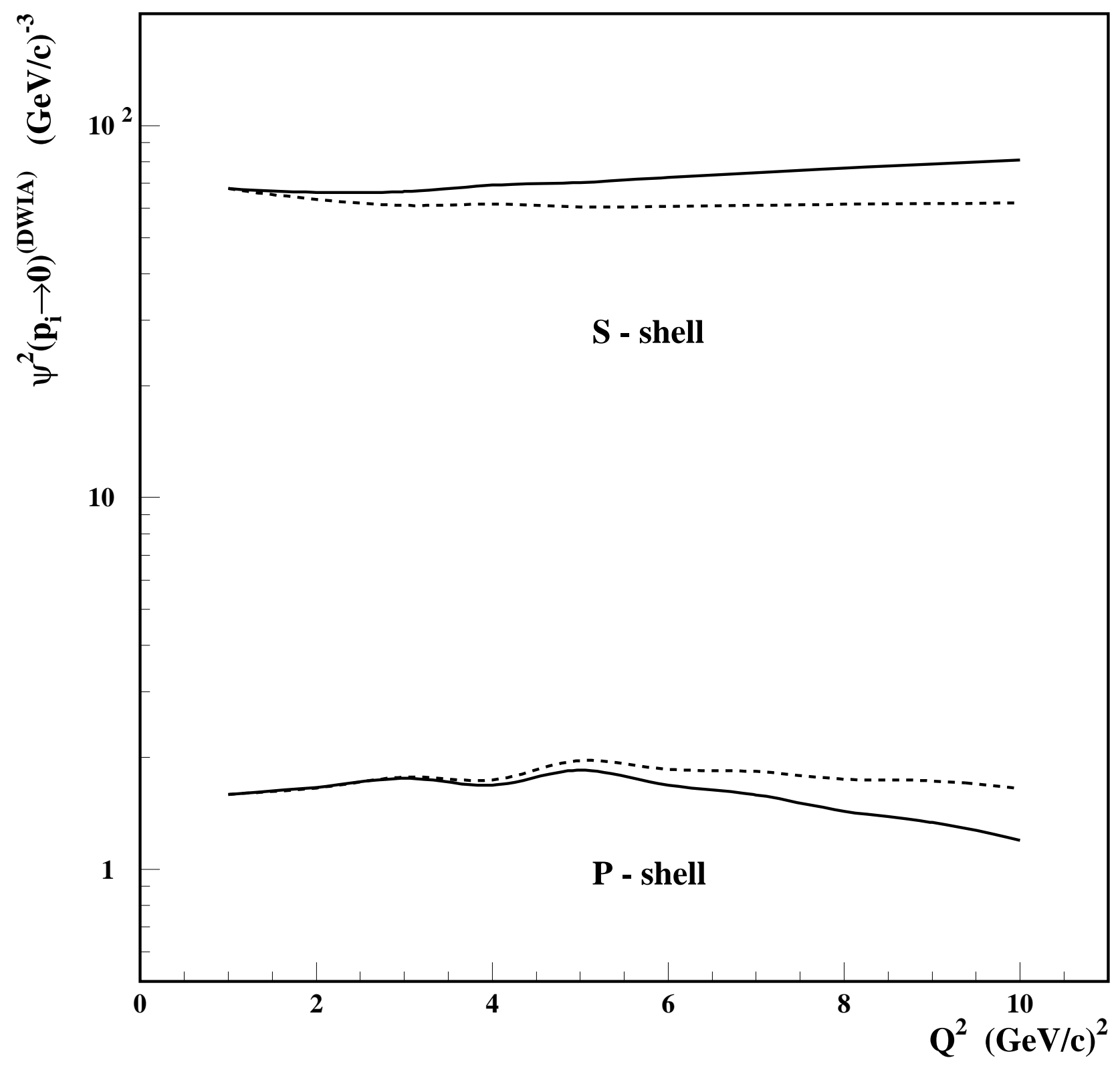

Fig.3 
This figure "fig1-4.png" is available in "png" format from: http://arxiv.org/ps/nucl-th/9501019v1 


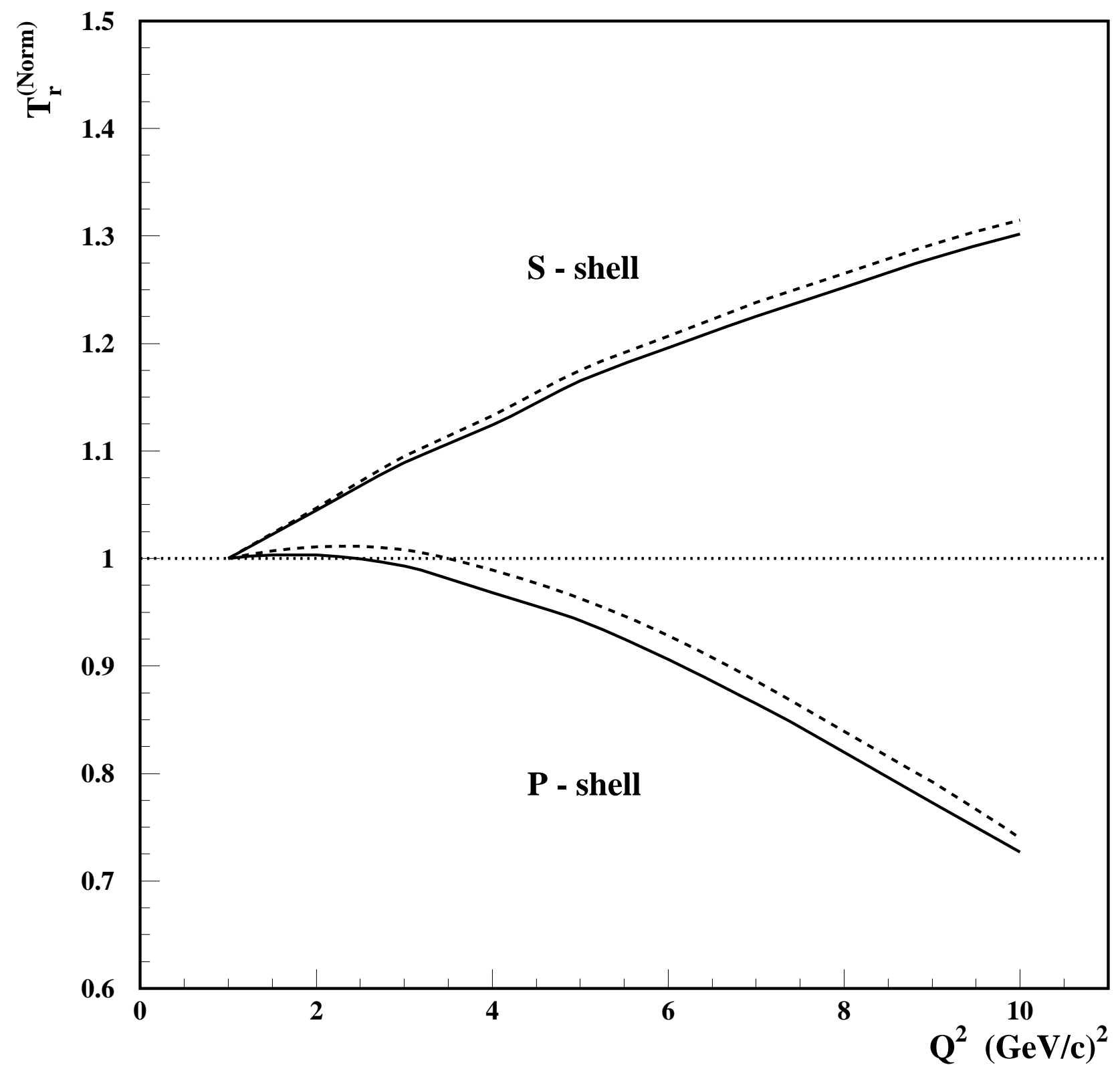

Fig.4 